Abstract 86 Table 1 Association between the different aPL and the number of positive antibodies and APS related features

\begin{tabular}{|c|c|c|c|c|c|c|}
\hline & Arterial thrombosis & Venous thrombosis & Small vessel thrombosis & Fetal death & $\geq 3$ pregnancy losses & Thrombocytopenia \\
\hline LA & $4.45(3.2-6.3) p<0.001$ & $4.9(3.8-6.3) p<0.001$ & $4.7(3.2-7) p<0.001$ & $1.7(1.2-2.5) p<0.001$ & $4.1(2.2-7.3) p<0.001$ & $2.3(1-9-2.8) p<0.001$ \\
\hline aB2GP IgM & $3.5(2.3-5.2) p<0.001$ & $1.8(1.3-2.3) p=0.001$ & $2.7(1.6-4.3) p<0.001$ & $2.2(1.4-3.5) p=0.001$ & $4.6(2.3-9) p<0.001$ & $1.7(1.3-2.2) p<0.001$ \\
\hline aB2GP IgG & $6.5(4.4-9.5) p<0.001$ & $3.2(2.3-4.4) p<0.001$ & $3.7(2.3-5.8) p<0.001$ & $1.8(1.1-2.9) \mathrm{p}=0.024$ & $5.2(2.7-10) p<0.001$ & $1.6(1.2-2.1) p=0.001$ \\
\hline $\mathrm{aCL} \lg \mathrm{M}$ & $2.4(1.8-3.4) p<0.001$ & $2.4(1.9-3.0) p<0.001$ & $4(2.8-5.8) p<0.001$ & $1.7(1.2-2.4) \mathrm{p}=0.006$ & $3.1(1.8-5.3) p<0.001$ & $1.8(1.5-2.1) p<0.001$ \\
\hline aCL IgG & $7.3(5.2-10.2) p<0.001$ & $4(3.2-5.0) p<0.001$ & $4(2.8-5.7) p<0.001$ & $1.9(1.4-2.6) p<0.001$ & $3.8(2.2-6.6) p<0.001$ & $1.8(1.5-2.1) p<0.001$ \\
\hline $\mathrm{N}^{\circ}$ of positive antibodies & $2.5(2.2-2.9) p<0.001$ & $2.2(1.9-2.4) p<0.001$ & $2.3(1.9-2.4) p<0.001$ & $1.4(1.2-1.7) p<0.001$ & $2.3(1.8-2.9) p<0.001$ & $1.4(1.3-1.6) p<0.001$ \\
\hline
\end{tabular}

Background Antiphospholipid antibodies (aPL) have been associated with organ damage and certain features in systemic lupus erythematosus (SLE) patients. Our aim is to investigate the association between the different aPL and SLE manifestations as well as to elucidate the influence of the load of antibodies.

Methods Patients from the RELESSER-T registry were included. RELESSER-T is a multicenter, hospital-based registry, with retrospective cross-sectional collection of data from a large representative sample of adult non-selected patients with SLE attending Spanish rheumatology services from the public national health system.

Results Out of a total of 3651 SLE patients, 1368 were positive for aPL (44.9\% of patients were positive for anticardiolipin (aCL) antibodies, $27.3 \%$ for anti b2glycoprotein I (aB2GPI) and 24\% for lupus anticoagulant (LA)). Regarding the load of antibodies, 20.6\%, 12.1\% and 4.8\% were positive for one, two and three antibodies, respectively. The association between the different aPL, the number of positive antibodies and antiphospholipid syndrome related manifestations is showed in table 1 . Overall, all types of aPL were associated with classic APS manifestations, although LA, IgG isotypes, and patients with more than one aPL display a higher risk to develop clinical APS.

Regarding specific lupus manifestations, all aPL types showed a negative association with cutaneous manifestations, and was also significantly associated with the load of autoantibodies $(\mathrm{p}<0.001)$. LA and aCL were associated with an increased risk of cardiac, respiratory and neuropsychiatric manifestations $(p<0.001)$. Furthermore, LA was also associated with an increased risk of renal disease $(p<0.001)$. aCL $\operatorname{IgG}$ was associated with a higher risk of specific lupus manifestations compared with aCL IgM. Interestingly, aB2GP IgG were only associated with an increased risk of seizures $(p<0.001)$. When evaluating the influence of the load of antibodies, we found that the risk of neuropsychiatric manifestations $(\mathrm{p}<0.001)$, as well as the cardiac $(\mathrm{p}=0.003)$, and pulmonary manifestations $(p=0.001)$, significantly increased with a higher number of positive antibodies. Inversely, the risk of cutaneous symptoms decreased while the number of positive antibodies increased (OR 0.89, 95\% CI 0.82-0.96, p=0.003).

Conclusions The present study in a large SLE cohort confirm than there is a hierarchy for aPL and the risk of APS and lupus manifestations. aCL, and especially LA, confer a higher risk for major organ involvement in SLE patients. IgG isotypes and the load of aPL antibodies confer a higher risk for clinical APS and major lupus manifestations.

Funding Source(s): None

\section{MAPPING DISEASE SEVERITY AND PROGRESSION OF RENAL INVOLVEMENT IN PATIENTS WITH SYSTEMIC LUPUS ERYTHEMATOSUS}

${ }^{1} J u s t y n a$ Amelio, ${ }^{2}$ Kerry Gairy, ${ }^{3}$ Anadi Mahajan, ${ }^{3}$ Gavneet Kaur, ${ }^{4}$ Damon Bass, ${ }^{4}$ Rodger Levy*, ${ }^{5}$ David Roth. 'GlaxoSmithKline, Real World Evidence and Epidemiology, Stevenage, Herts, UK; ${ }^{2}$ GlaxoSmithKline, Value Evidence and Outcomes, Brentford, Middlesex, UK; ${ }^{3}$ Bridge Medical, Richmond, London, UK; ${ }^{4}$ GlaxoSmithKline, Immunoinflammation and Future Pipeline, Collegeville, PA, USA; ${ }^{5}$ GlaxoSmithKline, Research and Development, Collegeville, PA, USA

\subsection{6/lupus-2019-Ism.87}

Background Despite recent advances in the treatment of systemic lupus erythematosus (SLE) and lupus nephritis (LN), understanding of their pathogenesis and the interrelation between disease states remains incomplete. A pragmatic review (GSK study LS3178) was conducted to map disease severity and progression of renal involvement in SLE, focusing on: LN development among patients with SLE, within-LN progression, and progression to end-stage renal disease (ESRD).

Methods A keyword based literature search was conducted using PubMed, Google and Google Scholar and supplemented with a bibliography search relevant to the focus area. The following publications were screened and prioritized for inclusion: high quality; published after 2010; addressed a topic of focus or an information gap; data were from the USA or Europe. High-quality pre-2010 and non-USA/Europe publications were permitted.

Results Overall, 248 citations were identified (keyword based search, $n=117$; bibliography search, $n=131$ ). Following full text screening, 144 publications were considered relevant to the review and 26 were selected for inclusion (21 primary studies, 3 narrative reviews and 2 systematic literature reviews). An overview of the results is provided in the Figure. This review identified that $726 \%$ of patients had LN at the time of SLE diagnosis, and $3148 \%$ of patients with SLE developed LN in the disease course, most (8090\%) within 5 years of diagnosis. Class IV nephritis was the most common LN class found at first (3560\%) and repeat (3563\%) biopsy and had the worst prognosis. Histological transformation from one LN class to another was reported in $4076 \%$ of patients, most commonly in patients with nonproliferative lesions in the first biopsy. Overall, the proportion of patients who subsequently developed ESRD was $36 \%$ (SLE) and $428 \%$ (LN). Limited data existed for time to progression within LN and from SLE/LN to ESRD, and for renal signs present before LN diagnosis. 


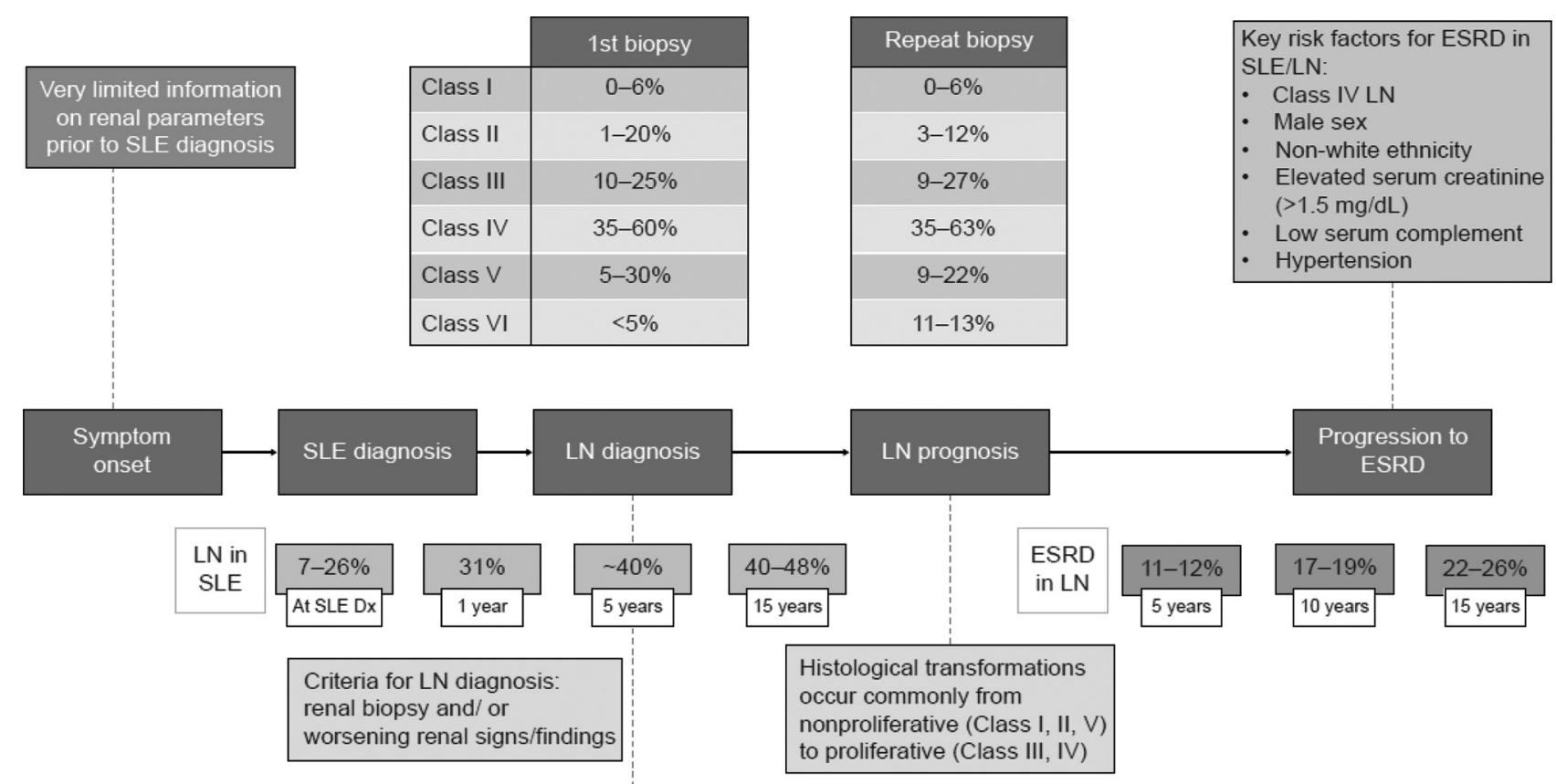

Risk factors for $\mathrm{LN}$ :

- Lower age at SLE diagnosis

- Male sex

- Being Hispanic, Asian or African

ESRD, end stage renal disease; LN, lupus nephritis; SLE, systemic lupus erythematosus; WHO, World Health Organization; Dx, diagnosis;

WHO classification (Class I = normal kidney; Class II = mesangial glomerulonephritis; Class III = focal segmental proliferative glomerulonephritis;

Class IV = diffuse proliferative glomerulonephritis; Class V = membranous glomerulonephritis; Class VI = chronic sclerosing glomerulonephritis)

Abstract 87 Figure 1

Conclusions This review highlights risk factors for developing LN and progressing from SLE/LN to ESRD. Male patients, patients of non white ethnicities, and patients of a younger age at SLE diagnosis had the highest risk for developing LN and progressing from SLE/LN to ESRD. Of the renal parameters, elevated serum creatinine was identified as the best predictor of worsening disease state. A higher risk of worse outcomes is seen in the earlier SLE/ LN disease stages, demonstrating the importance of early diagnosis and the need for effective disease modifying treatments for SLE and LN.

Funding Source(s): Study funded by GSK.

\section{ASSESSMENT OF CLINICAL CLASSIFICATION CRITERIA- BASED COMPUTATIONAL PHENOTYPES TO DETECT PATIENTS WITH SYSTEMIC LUPUS ERYTHEMATOSUS IN ELECTRONIC HEALTH RECORDS}

\footnotetext{
${ }^{1}$ Theresa Walunas*, ${ }^{1}$ Anika S Ghosh, ${ }^{1}$ Jennifer Pacheco, ${ }^{1}$ Kathryn Jackson, ${ }^{2}$ Anh H Chung, ${ }^{1}$ Daniel Erickson, ${ }^{2}$ Karen Mancera-Cuevas, ${ }^{1}$ Abel Kho, ${ }^{2}$ Rosalind Ramsey-Goldman. ${ }^{1}$ Northwestern University; ${ }^{2}$ Northwestern University, Feinberg School of Medicine
}

\subsection{6/lupus-2019-Ism.88}

Background Systemic Lupus Erythematosus (SLE) is a systemic autoimmune disease with diverse manifestations that can occur over a long period of time. Electronic health record (EHR) data presents a rich source of information that can be used to understand the varied presentation of SLE. We examined three clinical classification criteria for SLE to assess whether they could be a foundation for phenotype-based detection of patients with SLE in EHR data.

Methods We assessed algorithm performance over 600 medical records from the Northwestern Medicine Electronic Data Warehouse, 472 of which had definite SLE and 128 which did not, based on chart review. We developed algorithms, based on the American College of Rheumatology (ACR), Systemic Lupus International Collaborating Clinics (SLICC) and the proposed European League Against Rheumatism/American College of Rheumatology (EULAR/ACR) classification criteria using only structured data elements (diagnosis codes (ICD9/ICD10) and lab results) to determine whether patients met the classification criteria for definite SLE.

Results As shown in table 1 , the overall identification rate of SLE ranged from $58 \%$ to $78 \%$ across the three algorithms. All three criteria-based algorithms had greater than $95 \%$ specificity and greater than $98 \%$ positive predictive value (PPV). Sensitivity of the algorithms ranged from 52\% to $69 \%$ and negative predictive value (NPV) from $35 \%$ to $55 \%$. The SLICC-based algorithm had the overall highest performance, detecting $78 \%$ of the patients with definite SLE as determined by chart review, with 99\% PPV, 69\% sensitivity, 98\% specificity and 55\% NPV.

Conclusions The ACR-, SLICC- and proposed EULAR/ACRbased EHR algorithms all detect a significant proportion of patients that were classified as having definite SLE by chart review, with high PPV and specificity. Low NPV of 